\title{
ATIVIDADE RIZOSFÉRICA DE SOLO TRATADO COM HERBICIDA DURANTE PROCESSO DE REMEDIAÇÃO POR Stizolobium aterrimum ${ }^{1}$
}

\author{
Edson Aparecido dos Santos ${ }^{2}$, Mauricio Dutra Costa ${ }^{3}$, Lino Roberto Ferreira ${ }^{4}$, \\ Marcelo Rodrigues dos Reis ${ }^{5}$, André Cabral França ${ }^{6}$, José Barbosa dos Santos ${ }^{6}$
}

\section{ABSTRACT \\ RHIZOSPHERE ACTIVITY OF SOIL \\ TREATED WITH HERBICIDE DURING REMEDIATION PROCESS BY Stizolobium aterrimum}

This study aimed to evaluate the soil microbiota activity associated to $S$. aterrimum rhizosphere, during the phytoremediation process of a soil contaminated with trifloxysulfuron-sodium. S. aterrimum was cultivated in vases $(6,0 \mathrm{~L})$ filled with soil, after trifloxysulfuron-sodium, at $7.5 \mathrm{~g} \mathrm{ha}^{-1}$, was applied or not. Every 15 days after sowing (DAS), rhizospheric soil was collected for soil microbial respiration (amount of evolved $\mathrm{CO}_{2}$ ) and microbial biomass carbon (MBC) analysis. To determine herbicide residues in the soil samples, after phytoremediation, Sorghum bicolor L. was cultivated as an indicator species, in addition to high performance liquid chromatography (HPLC) analysis. The microbiota associated to $S$. aterrimum rhizosphere showed to be sensitive to trifloxysulfuron-sodium and may be used as a microbiological indicator of disturbances caused by this herbicide in the environment. Regarding time for remediation, 45 days of development is enough for $S$. aterrimum to reduce residual trifloxysulfuron-sodium to a level not able to cause toxicity to the indicator plant.

KEY-WORDS: Microbiological indicator; phytoremediation; microbial biomass.

\section{INTRODUÇÃO}

A fitorremediação é o uso de plantas e sua microbiota associada na descontaminação de solo e água, explorando a habilidade de algumas espécies vegetais de remover, extrair e/ou mineralizar xenobióticos, bem como a tolerância dessas plantas aos

\section{RESUMO}

Objetivou-se, com este trabalho, avaliar a atividade da microbiota associada à rizosfera de Stizolobium aterrimum, durante processo de fitorremediação de solo contaminado com o herbicida trifloxysulfuron-sodium. Em vasos com capacidade para seis litros, após aplicação ou não de 7,5 $\mathrm{g} \mathrm{ha}^{-1} \mathrm{do}$ herbicida trifloxysulfuron-sodium, cultivou-se a espécie vegetal S. aterrimum. Após a semeadura, procedeu-se à coleta de solo rizosférico, quinzenalmente, para determinação da respiração da microbiota do solo (quantidade de $\mathrm{CO}_{2}$ evoluído) e carbono da biomassa microbiana (CBM). Para determinação do resíduo do herbicida nas amostras de solo, após a remediação, cultivou-se sorgo (Sorghum bicolor L.), como espécie bioindicadora, além de ser efetuada análise por cromatografia líquida de alta eficiência (CLAE). Observou-se que a microbiota associada à rizosfera de $S$. aterrimum é sensível ao trifloxysulfuron-sodium, constituindose indicador microbiológico de distúrbios causados por este herbicida no ambiente. Quanto ao tempo para remediação, constatou-se que 45 dias de desenvolvimento é tempo suficiente para que $S$. aterrimum promova a diminuição do resíduo de trifloxysulfuron-sodium a um nível incapaz de causar toxicidade à planta indicadora.

PALAVRAS-CHAVE: Indicador microbiológico; fitorremediação; biomassa microbiana.

contaminantes (Wilson et al. 2000). Essa técnica apresenta-se como alternativa agronômica para sistemas de cultivo nos quais há a necessidade de se remover herbicidas de alta persistência no solo, como o trifloxysulfuron-sodium. Este herbicida é utilizado na cultura do algodoeiro (Gossypium hirsutum L.), em pós-emergência inicial, ou em mistura ao ametryn,

1. Trabalho recebido em set./2008 e aceito para publicação em mar./2010 (nº registro: PAT 4670/ DOI: 10.5216/pat.v40i1.4670).

2. Universidade Estadual Paulista, Campus Jaboticabal, Departamento de Produção Vegetal, Jaboticabal, SP, Brasil.

E-mail: edsonsantos@posgrad.fcav.unesp.br.

3. Universidade Federal de Viçosa, Centro de Ciências Biológicas, Departamento de Microbiologia, Viçosa, MG, Brasil. E-mail:mdcosta@ufv.br.

4. Universidade Federal de Viçosa, Centro de Ciências Agrárias, Departamento de Fitotecnia, Viçosa, MG, Brasil. E-mail:1roberto@ufv.br.

5. Universidade Federal do Tocantins, Campus Gurupi, Departamento de Produção Vegetal, Gurupi, TO, Brasil. E-mail: reisagro@yahoo.com.br.

6. Universidade Federal dos Vales do Jequetinhonha e Mucuri, Campus Diamantina, Departamento de Agronomia, Diamantina, MG, Brasil.E-mails: cabralfranca@yahoo.com.br, jbarbosa@ufvjm.edu.br. 
na cana-de-açúcar (Saccharum officinarum L.) (Rodrigues \& Almeida 2005), apresentando problemas de carryover, em culturas sensíveis plantadas até oito meses após aplicação, sendo, porém, passíveis de remediação pela mucuna-preta (Stizolobium aterrimum) (Procópio et al. 2005).

Pode-se afirmar que a fitorremediação é uma técnica aceleradora da retirada de compostos tóxicos do solo (no caso, herbicidas), promovendo sua descontaminação (Cunningham et al. 1996). Baseiase na seletividade que algumas espécies exibem a um composto específico e/ou a um mecanismo de ação. A tolerância pode resultar de processos como a translocação diferencial de compostos orgânicos para outros tecidos da planta, com subsequente volatilização, ou degradação parcial ou completa transformação em compostos menos tóxicos, combinados e/ou ligados aos tecidos das plantas (Accioly \& Siqueira 2000). Além disso, pode ocorrer a fitoestimulação, devido à liberação de exsudatos radiculares, o que estimula a atividade microbiana responsável pela degradação de um composto no solo, caracterizando, em algumas plantas, a aptidão rizosférica para a biorremediação de compostos tóxicos (Cunningham et al. 1996, Accioly \& Siqueira 2000, Scramin et al. 2001).

Em vários trabalhos, é relatada a contribuição das plantas, estimulando, por meio do efeito rizosférico, a mineralização de alguns herbicidas, principalmente atrazine e metolachlor (Anderson et al. 1994, Anderson \& Coats 1995, Perkovich et al. 1996). Destaca-se o trabalho realizado por Burken \& Schnoor (1996), que, objetivando avaliar o potencial remediador de árvores, por meio de exsudação de substâncias e posterior estímulo à microbiota, observaram que a espécie Populus deltoides foi capaz de absorver a maior parte de atrazine aplicada, sem desenvolver sintomas de fitotoxidez.

Pela avaliação da atividade microbiana na rizosfera, pode-se inferir sobre o papel da microbiota, quando estimulada pelas raízes das plantas, na remediação dos herbicidas. Essa atividade pode ser estimada, indiretamente, por diferentes variáveis, tais como respiração basal do solo rizosférico (Paul et al. 1999), biomassa microbiana (Jenkinson \& Ladd 1981, Stenberg 1999), quociente metabólico $\left(\mathrm{qCO}_{2}\right)$ (Anderson \& Domsch 1985), porcentagem de colonização das raízes por fungos micorrízicos, número e atividade de nódulos na fixação de $\mathrm{N}_{2}$, no caso de leguminosas.
Vários fatores afetam os microorganismos associados à rizosfera, podendo, também, afetar o processo de fitorremediação. Dentre estes, podem ser citados os seguintes: características do solo, como teor de argila e matéria orgânica, $\mathrm{pH}$, disponibilidade de elementos e aeração (Tauk 1990); e características das plantas, como espécie, vigor, profundidade do sistema radicular, número e tamanho de raízes, quantidade de exsudados, materiais solúveis, mucigel, mucilagem e células mortas produzidas (Moreira \& Siqueira 2002). A exsudação de compostos orgânicos varia muito, em função da espécie. Segundo Newman \& Watson (1977), plantas de alfafa e de pinus exsudam maior volume de carboidratos, em relação a plantas de cevada e milho, e plantas de milho exsudam maior volume de aminoácidos, em relação a plantas de Robinia pseudoacacia (Fabaceae).

Outro fator importante, na relação planta $\mathrm{x}$ microorganismo de solo, é a idade da planta. Segundo Rovira (1965), em fase de florescimento, há maior liberação de exsudados radiculares, contribuindo para maior número de microorganismos associados à rizosfera. A deposição de materiais orgânicos (açúcares, ácidos orgânicos, aminoácidos e carboidratos) pelas plantas também é afetada pela idade das mesmas (Balarsubramanian \& Rangaswami 1969, Hale et al. 1971).

Diante do exposto, objetivou-se, com este trabalho, avaliar a atividade microbiana rizosférica de S. aterrimum, durante processo de fitorremediação de solo contaminado com o herbicida trifloxysulfuronsodium, identificando estádios onde a descontaminação é mais eficiente.

\section{MATERIAL E MÉTODOS}

O experimento foi conduzido em casa de vegetação e laboratório, pertencentes ao Departamento de Fitotecnia da Universidade Federal de Viçosa. Os tratamentos foram organizados em esquema fatorial $4 \times 5$ e compostos pela combinação de quatro sistemas de cultivo (A - solo contaminado com herbicida e cultivado com mucuna-preta; B - solo sem herbicida e cultivado com mucuna-preta; $\mathrm{C}$ - solo contaminado com herbicida e sem cultivo; e D - solo sem herbicida e sem cultivo) e cinco épocas de coleta (15,30, 45, 60 e 75 dias após a aplicação do herbicida - DAA). Cada tratamento contou com três repetições. Vasos com capacidade para 6,0 L foram preenchidos com $5 \mathrm{~kg}$ de terra fina seca ao ar (TFSA), coletada na região 
de Viçosa, MG (2046'S e 4252’W, com altitude de $650 \mathrm{~m}$ ), oriunda de um Argissolo vermelho-amarelo (Embrapa 2006), com as seguintes características físicas e químicas: matéria orgânica $=2,18 \mathrm{dag} \mathrm{kg}^{-1}$; argila $=39 \mathrm{dag} \mathrm{kg}^{-1}$; silte $=11 \mathrm{dag} \mathrm{kg}^{-1}$; areia fina $=$ 17 dag $\mathrm{kg}^{-1} \mathrm{e}$ areia grossa $=33 \mathrm{dag} \mathrm{kg}^{-1} ; \mathrm{P}($ resina $)=$ $0,6 \mathrm{mg} \mathrm{dm}^{-3} ; \mathrm{K}=18 \mathrm{mg} \mathrm{dm}^{-3} ; \mathrm{H}+\mathrm{Al}=5,9 \mathrm{cmolc} \mathrm{dm}^{-3}$; $\mathrm{Al}^{3+}=1,1 \mathrm{cmolc} \mathrm{dm}^{-3} ; \mathrm{Ca}^{2+}=0,1 \mathrm{cmolc} \mathrm{dm}^{-3} ; \mathrm{Mg}^{2+}=$ $0,0 \mathrm{cmolc} \mathrm{dm}^{-3}$; CTC total $=6,09 \mathrm{cmolc} \mathrm{dm}^{-3}$; saturação por bases $(\mathrm{V})=2,0 \%$; e saturação por alumínio $(\mathrm{m})=88 \%$.

A terra dos vasos foi umedecida para a aplicação do herbicida trifloxysulfuron-sodium. Foi utilizada a formulação comercial de grânulos dispersíveis, na dose de 7,5 $\mathrm{g} \mathrm{ha}^{-1}$, com volume de calda de $200 \mathrm{~L} \mathrm{ha}^{-1}$. A aplicação foi realizada de forma dirigida, com pulverizador costal pressurizado com $\mathrm{CO}_{2}$, equipado com barra de dois metros e duas pontas de pulverização do tipo leque 110.015 . Após 24 horas da aplicação, realizou-se a semeadura de três sementes de mucuna-preta (Stizolobium aterrimum) por vaso. Após a emergência e estabelecimento das plântulas, realizou-se desbaste, mantendo-se uma planta por vaso.

Para cada época de avaliação, foi retirada uma amostra da terra de preenchimento dos vasos (aproximadamente 300 gramas), que, após ser passada por peneira de 2 mesh, seca ao ar e ter sua umidade determinada, foi pesada e adicionada a frascos Erlenmayer. Cada recipiente recebeu o equivalente a $100 \mathrm{~g}$ de solo seco, sendo o teor de água, nas amostras, mantido em $70 \%$ da capacidade de campo.

Nas épocas pré-estabelecidas, avaliou-se a respiração da microbiota do solo, nos tratamentos, por meio da quantificação do $\mathrm{CO}_{2}$ evoluído. Para isso, $100 \mathrm{~mL}$ de solução de $\mathrm{NaOH}\left(0,25 \mathrm{~mol} \mathrm{~L}^{-1}\right)$ foram acondicionados em frascos de vidros e mantidos em sistema contínuo de fluxo de ar (isento de $\mathrm{CO}_{2} \mathrm{e}$ umidade), para apreensão do $\mathrm{CO}_{2}$ evoluído. $\mathrm{O} \mathrm{NaOH}$ em excesso foi determinado por titulação com $\mathrm{HCl}$ $\left(0,25 \mathrm{~mol} \mathrm{~L}^{-1}\right)$, permitindo, assim, a determinação da quantidade de $\mathrm{CO}_{2}$ evoluído das amostras incubadas (Jenkinson \& Powlson 1976).

Em cada avaliação, foi determinado, também, o carbono da biomassa microbiana (CBM), pelo método descrito por Vance et al. (1987), utilizandose, em lugar do clorofórmio (fumigação), forno de microondas (irradiação) (Islam \& Weil 1998).

Para verificação do residual herbicida, nas amostras de solo, cultivou-se sorgo (Sorghum bicolor L.), como espécie indicadora, no bioensaio (Vivian et al. 2007). Em vasos com volume de 0,2 L, foram colocados 150 gramas de solo proveniente de subamostras retiradas em cada época e semearamse três sementes de sorgo, deixando-se apenas duas plantas por vaso. Após desenvolvimento, as plantas foram avaliadas, aos 25 dias após o semeio. Procedeuse, também, à análise cromatográfica, submetendo-se subamostras de terra à agitação rotacional, centrifugação e coleta do sobrenadante filtrado. Para quantificação da concentração do herbicida em solução, uma alíquota, previamente filtrada, foi analisada por Cromatografia Líquida de Alta Eficiência (CLAE), em aparelho Shimandzu, equipado com detector UV, a $245 \mathrm{~nm}$, e coluna Varian de fase reversa (C-18). A fase móvel foi composta por acetonitrila, água e ácido fosfórico, na respectiva proporção 48:52:1 (v:v:v), com fluxo de $1 \mathrm{~mL} \mathrm{~min}^{-1}$. Utilizou-se o método do padrão externo, em solução de $\mathrm{CaCl}_{2} 0,01 \mathrm{~mol} \mathrm{~L}^{-1}$.

Após a coleta e tabulação dos dados, os mesmos foram submetidos a análise de variância. $\mathrm{O}$ efeito do intervalo sobre as características avaliadas, após a semeadura, foi decomposto em linear, quadrático, raiz quadrada, cúbico, hiperbólico e logístico, obtendo-se, em seguida, as equações de regressão com maior coeficiente de determinação e que melhor representavam o fenômeno biológico. As estimativas dos parâmetros dos modelos foram testadas pelo teste t, a $1 \%$ e $5 \%$ de probabilidade de erro. Entre os tratamentos, com ou sem herbicida, e planta, realizou-se comparação de médias, pelo teste Tukey, com 5\% de probabilidade.

\section{RESULTADOS E DISCUSSÃO}

Em todas as épocas de avaliação, observou-se excelente desenvolvimento da mucuna-preta, independentemente da aplicação do herbicida, confirmando a tolerância dessa espécie ao trifloxysulfuronsodium, conforme já descrito em outros trabalhos (Procópio et al. 2005, Santos et al. 2005).

Observou-se comportamento crescente para valores de acúmulo de $\mathrm{CO}_{2}$, ao longo do período de incubação das amostras de solo, sob os diferentes tratamentos. O maior acúmulo de $\mathrm{CO}_{2}$ foi observado para o solo cultivado com mucuna-preta, quando não houve aplicação do herbicida. Menor atividade metabólica, evidenciada pelo desprendimento de $\mathrm{CO}_{2}$, foi verificada no solo não cultivado e contaminado com trifloxysulfuron-sodium (Tabela 1). 
Tabela 1. Total do $\mathrm{CO}_{2}$ acumulado, proveniente da microbiota do solo contaminado com o herbicida trifloxysulfuron-sodium e remediado ou não por Stizolobium aterrimum (Viçosa, MG, 2008).

\begin{tabular}{|c|c|c|c|c|}
\hline \multirow{3}{*}{$\mathrm{DAS}^{1}$} & \multicolumn{4}{|c|}{$\mathrm{CO}_{2}$ acumulado $\left(\mu \mathrm{g} \mathrm{g}^{-1}\right)$} \\
\hline & \multicolumn{2}{|c|}{ Com $S$. aterrimum } & \multicolumn{2}{|c|}{ Sem $S$. aterrimum } \\
\hline & Sem herbicida & Com herbicida & Sem herbicida & Com herbicida \\
\hline 15 & $1705 \mathrm{~A}^{2}$ & $1485 \mathrm{~A}$ & $1430 \mathrm{~A}$ & $1412 \mathrm{~A}$ \\
\hline 30 & $2567 \mathrm{~A}$ & $2310 \mathrm{~A}$ & $2108 \mathrm{~A}$ & 1999 A \\
\hline 45 & 3932 A & $3510 \mathrm{AB}$ & $3327 \mathrm{AB}$ & $3181 \mathrm{~B}$ \\
\hline 60 & $4856 \mathrm{~A}$ & $4345 \mathrm{AB}$ & $4363 \mathrm{AB}$ & 3887 B \\
\hline 75 & $5903 \mathrm{~A}$ & $5225 \mathrm{AB}$ & $5317 \mathrm{~A}$ & $4565 \mathrm{~B}$ \\
\hline Equação $^{3}$ & $\hat{\mathrm{Y}}=71,231 \mathrm{D} *+587,111$ & $\hat{\mathrm{Y}}=63,434 \mathrm{D} *+520,521$ & $\hat{\mathrm{Y}}=66,865 \mathrm{D} *+300,298$ & $\hat{\mathrm{Y}}=54,622 \mathrm{D}^{*}+550,577$ \\
\hline
\end{tabular}

A respiração total da microbiota da amostra de solo não contaminado e não cultivado foi semelhante aos valores observados para as amostras provenientes da rizosfera de mucuna, em solo previamente contaminado pelo herbicida (Tabela 1). É provável que a maior atividade observada no solo contaminado com herbicida e, posteriormente, cultivado com a mucuna-preta se deva à maior concentração de microorganismos e abundância de exsudados provenientes do desenvolvimento da espécie vegetal. Pires et al. (2005) observaram que o solo rizosférico tratado com tebuthiuron apresentou maior desprendimento de $\mathrm{CO}_{2}$, se comparado ao solo não vegetado, sendo este fato atribuído à fitoestimulação da microbiota associada à rizosfera.

Considerando-se a complexidade do sistema radicular em solo, quanto à possibilidade de interações com microorganismos e à variedade de compostos presentes, várias são as possibilidades de quebra de compostos xenobióticos, por meio da rizodegradação, mecanismo que contribui, de maneira integrada, à fitoestimulação (Santos et al. 2007). Nesse sentido, a variedade de compostos, bem como o estádio fenológico e a idade da planta, podem garantir maior sucesso no processo de descontaminação. Richter et al. (1968), Balarsubramanian \& Rangaswami (1969) e Hale et al. (1971) mostraram que a exsudação de $\mathrm{N}$-amina, em raízes de sorgo, decresce com a idade da planta e observaram, também, maior exsudação de açúcares. Para a Crotalaria juncea, esses mesmos autores encontraram valores de exsudação de $\mathrm{N}$-amina maiores, aos 30 dias após a emergência das plantas, em relação a 15 e 45 dias.

A avaliação do total de carbono proveniente da biomassa microbiana das amostras de solo evidenciou maior valor para o solo cultivado com mucuna-preta, independentemente da aplicação do trifloxysulfuronsodium, contudo, sem diferença significativa para o solo sem cultivo prévio e sem herbicida (Tabela 2). O menor valor de CBM $\left(136,25 \mu \mathrm{g} \mathrm{g}^{-1}\right)$ foi observado para o solo tratado com o herbicida e não cultivado, previamente, com mucuna-preta (Tabela 2). Acreditase que a adição do trifloxysulfuron-sodium, em solo não vegetado, promove efeito negativo à biomassa microbiana não beneficiada pelo efeito rizosférico. Vários outros trabalhos relatam o efeito negativo de herbicidas a microorganismos do solo (Edwards 1989, Wardle 1994, Perschbacher et al. 1997, Kinney

Tabela 2. Carbono da biomassa microbiana (CBM) e quociente metabólico $\left(\mathrm{qCO}_{2}\right)$, em amostras de solo provenientes ou não da rizosfera de mucuna-preta, após aplicação ou não de 7,5 $\mathrm{g} \mathrm{ha}^{-1}$ de trifloxysulfuron-sodium, coletadas aos 45 dias após a semeadura (Viçosa, MG, 2008).

\begin{tabular}{cccccc}
\hline \multirow{2}{*}{ Característica } & \multicolumn{2}{c}{ Com S. aterrimum } & \multicolumn{2}{c}{ Sem S. aterrimum } \\
\cline { 2 - 5 } & Sem herbicida & Com herbicida & Sem herbicida & Com herbicida \\
\hline $\mathrm{CBM}\left(\mu \mathrm{g} \mathrm{g}^{-1}\right)$ & $281,89 \mathrm{~A}^{*}$ & $258,34 \mathrm{~A}$ & 219,87 & $\mathrm{AB}$ & $136,25 \mathrm{~B}$ \\
$\mathrm{qCO}_{2}$ & $0,031 \mathrm{~A}$ & $0,030 \mathrm{~A}$ & $0,034 \mathrm{AB}$ & $0,052 \mathrm{~B}$ \\
\hline
\end{tabular}

* Médias seguidas por letras iguais, na linha, não diferem entre si pelo teste Tukey, a $5 \%$ de probabilidade. 
et al. 2005) e o benefício da vegetação na atividade microbiana (Santos et al. 2006, 2007).

Uma estimativa mais representativa dos efeitos de qualquer estresse sobre a microbiota do solo pode ser obtida por meio da avaliação do quociente metabólico $\left(\mathrm{qCO}_{2}\right)$, o qual relaciona o $\mathrm{CO}_{2}$ acumulado e o total do CBM (Anderson \& Domsch 1985). Esse índice estabelece que, à medida que a biomassa microbiana se torna mais eficiente em utilizar os recursos em seu meio, menos $\mathrm{C}$ é perdido como $\mathrm{CO}_{2}$ pela respiração, podendo ele ser reincorporado aos tecidos microbianos. Dessa forma, menor $\mathrm{qCO}_{2}$ significa maior estabilidade da $\mathrm{BM}$ e, consequentemente, da matéria orgânica no solo. $\mathrm{O}$ valor de $\mathrm{qCO}_{2}$ foi maior nas amostras provenientes do solo tratado com o herbicida e não cultivado com mucuna-preta (Tabela 2). Dessa maneira, pode-se inferir, para esse tratamento, menor eficiência na utilização de $\mathrm{C}$, no metabolismo microbiano. Para os demais tratamentos, não se observaram diferenças no valor de $\mathrm{qCO}_{2}$.
A partir do bioensaio, foi possível comprovar a eficiência da mucuna-preta na descontaminação do solo com resíduo do trifloxysulfuron-sodium, observando-se intoxicação nas plantas de sorgo somente quando cultivadas em solo contaminado pelo herbicida e não cultivado pela mucuna-preta (Tabela 3). À exceção da primeira época de coleta de solo (30 DAS), para intoxicação visual, houve desenvolvimento do sorgo de maneira semelhante à testemunha, em todas as parcelas previamente cultivadas com mucuna-preta.

Durante o período vegetativo, a mucuna-preta desenvolve-se por, aproximadamente, 80 dias, sendo, após este período, cortada e incorporada ao solo, ou ainda deixada na superfície. Considerando-se os resultados obtidos, o período vegetativo até 45 dias de desenvolvimento da mucuna-preta é suficiente para descontaminação de solos com residual de trifloxysulfuron-sodium, na dose comercialmente recomendada de $7,5 \mathrm{~g} \mathrm{ha}^{-1}$. Favero et al. (2001), em

Tabela 3. Intoxicação visual (IV), altura e matéria seca da parte aérea (MSPA) de plantas de sorgo, em solo proveniente ou não da rizosfera de Stizolobium aterrimum, após tratamento ou não com o herbicida trifloxysulfuron-sodium $\left(7,5 \mathrm{~g} \mathrm{ha}^{-1}\right) \mathrm{e}$ coletado em quatro épocas distintas (Viçosa, MG, 2008).

\begin{tabular}{|c|c|c|c|c|}
\hline \multirow{3}{*}{$\mathrm{DAS}^{1}$} & \multicolumn{4}{|c|}{ IV $(\%)$} \\
\hline & \multicolumn{2}{|c|}{ Com S. aterrimum } & \multicolumn{2}{|c|}{ Sem S. aterrimum } \\
\hline & Sem herbicida & Com herbicida & Sem herbicida & Com herbicida \\
\hline 30 & $0,0 \mathrm{~B}^{2}$ & $10,0 \mathrm{~A}$ & $0,0 \mathrm{~B}$ & $90 \mathrm{~A}$ \\
\hline 45 & $0,0 \mathrm{~B}$ & $0,0 \mathrm{~B}$ & $0,0 \mathrm{~B}$ & $70 \mathrm{~A}$ \\
\hline 60 & $0,0 \mathrm{~B}$ & $0,0 \mathrm{~B}$ & $0,0 \mathrm{~B}$ & $66 \mathrm{~A}$ \\
\hline 75 & $0,0 \mathrm{~B}$ & $0,0 \mathrm{~B}$ & $0,0 \mathrm{~B}$ & $70 \mathrm{~A}$ \\
\hline Equação $^{3}$ & $\hat{\mathrm{Y}}=0,00$ & $\hat{\mathrm{Y}}=2,50$ & $\hat{\mathrm{Y}}=0,00$ & $\hat{\mathrm{Y}}=74,00$ \\
\hline \multirow{3}{*}{ DAS } & \multicolumn{4}{|c|}{ Altura $(\mathrm{cm})$} \\
\hline & \multicolumn{2}{|c|}{ Com $S$. aterrimum } & \multicolumn{2}{|c|}{ Sem S. aterrimum } \\
\hline & Sem herbicida & Com herbicida & Sem herbicida & Com herbicida \\
\hline 30 & $28,33 \mathrm{~A}$ & $22,67 \mathrm{~B}$ & $29,33 \mathrm{~A}$ & $12,33 \mathrm{~B}$ \\
\hline 45 & $33,00 \mathrm{~A}$ & $28,00 \mathrm{~A}$ & $33,67 \mathrm{~A}$ & $13,33 \mathrm{~B}$ \\
\hline 60 & $30,33 \mathrm{~A}$ & $29,67 \mathrm{~A}$ & $29,33 \mathrm{~A}$ & $12,87 \mathrm{~B}$ \\
\hline 75 & $30,33 \mathrm{~A}$ & $28,33 \mathrm{~A}$ & $30,00 \mathrm{~A}$ & $13,67 \mathrm{~B}$ \\
\hline Equação & $\hat{Y}=30,50$ & $\hat{\mathrm{Y}}=27,17$ & $\hat{\mathrm{Y}}=30,58$ & $\hat{\mathrm{Y}}=13,05$ \\
\hline \multirow{3}{*}{ DAS } & \multicolumn{4}{|c|}{$\operatorname{MSPA}(\mathrm{g})$} \\
\hline & \multicolumn{2}{|c|}{ Com $S$. aterrimum } & \multicolumn{2}{|c|}{ Sem $S$. aterrimum } \\
\hline & Sem herbicida & Com herbicida & Sem herbicida & Com herbicida \\
\hline 30 & $0,91 \mathrm{~A}$ & $0,67 \mathrm{~B}$ & $0,96 \mathrm{~A}$ & $0,18 \mathrm{~B}$ \\
\hline 45 & $0,98 \mathrm{~A}$ & $0,92 \mathrm{~A}$ & $0,89 \mathrm{~A}$ & $0,26 \mathrm{~B}$ \\
\hline 60 & $0,96 \mathrm{~A}$ & $0,91 \mathrm{~A}$ & $0,92 \mathrm{~A}$ & $0,33 \mathrm{~B}$ \\
\hline 75 & $1,00 \mathrm{~A}$ & $0,98 \mathrm{~A}$ & $0,97 \mathrm{~A}$ & $0,32 \mathrm{~B}$ \\
\hline Equação & $\hat{\mathrm{Y}}=0,96$ & $\hat{\mathrm{Y}}=0,87$ & $\hat{\mathrm{Y}}=0,94$ & $\hat{\mathrm{Y}}=0,27$ \\
\hline
\end{tabular}

${ }^{1}$ Dias após a semeadura de $S$. aterrimum.

${ }^{2}$ Médias seguidas por letras iguais, em cada linha, não diferem entre si pelo teste Tukey, a $5 \%$ de probabilidade de erro.

${ }^{3}$ Valores médios, sem significância, segundo teste t, a $5 \%$ de probabilidade de erro. 
trabalho visando a avaliar a capacidade de cobertura do solo por espécies de adubação verde, mostraram que, logo aos 28 dias após a emergência, plantas de mucuna-preta conferiram a maior cobertura do solo. Nesse sentido, Procópio et al. (2005) relatam que apenas 25 plantas $\mathrm{m}^{2}$ são suficientes para descontaminar solo com residual de trifloxysulfuron-sodium, para cultivo de feijão.

O bioensaio foi fundamental para avaliação dos resíduos do herbicida em estudo, pois conseguiu detectar níveis não observados pela cromatografia. A análise das amostras em cromatografia líquida de alta eficiência (CLAE) impossibilitou a determinação do nível de resíduos do herbicida presente no solo, tendo em vista que a dose do trifloxysulfuronsodium comercialmente recomendada $\left(7,5 \mathrm{~g} \mathrm{ha}^{-1}\right)$ ficou abaixo do limite de quantificação (LQ) do método CLAE. Em estudos realizados por Vivian (2007), a extração e avaliação dos resíduos de trifloxysulfuron-sodium, em diferentes solos, permitiram quantificação segura desse herbicida, para valores de LQ superiores a $0,08 \mu \mathrm{g} \mathrm{kg}^{-1} \mathrm{de}$ solo. Neste trabalho, considerando-se o volume de solo utilizado, a concentração do trifloxysulfuronsodium foi de, aproximadamente, $0,004 \mu \mathrm{g} \mathrm{kg}^{-1} \mathrm{de}$ solo, ou seja, 20 vezes abaixo do LQ estabelecido por Vivian (2007). Apesar da baixa concentração no solo, esse nível de contaminação é suficiente, em nível de campo, para intoxicação de espécies sensíveis cultivadas.

\section{CONCLUSÕES}

1. A microbiota associada à rizosfera da mucuna$\operatorname{preta}(S$. aterrimum) é sensível ao trifloxysulfuronsodium, podendo constituir-se em indicador microbiológico de distúrbios causados por este herbicida no ambiente.

2. Com 45 dias de crescimento e desenvolvimento da mucuna-preta, o resíduo do herbicida trifloxysulfuron-sodium é reduzido a um nível, no solo, não capaz de causar toxicidade à planta indicadora.

\section{AGRADECIMENTOS}

Ao Conselho Nacional de Desenvolvimento Científico e Tecnológico $(\mathrm{CNPq})$, pelo apoio financeiro à realização deste trabalho.

\section{REFERÊNCIAS}

ACCIOLY, A. M. A.; SIQUEIRA, J. O. Contaminação química e biorremediação do solo. In: NOVAIS, R. F.; ALVAREZ, V. H.; SCHAEFER, C. E. G. R. (Eds.). Tópicos em ciência do solo. Viçosa: Sociedade Brasileira de Ciência do Solo, 2000. p. 299-352.

ANDERSON, T. A.; COATS, J. R. Screening rhizosphere soil samples for the ability to mineralize elevated concentrations of atrazine and metolachlor. Journal of Environment Science and Health, Ames, v. 30, n. 4, p. 473-484, 1995.

ANDERSON, T. A.; KRUGER, E. L.; COATS, J. R. Enhanced degradation of a mixture of three herbicides in the rhizosphere of a herbicide-tolerant plant. Chemosphere, Oxford, v. 28, n. 8, p. 1551-1557, 1994.

ANDERSON, T. H.; DOMSCH, K. H. Determination of ecophysiological maintenance carbon requirements of soil microorganisms in a dormant state. Biology and Fertility of Soils, Firenze, v. 1, n. 2, p. 81-89, 1985.

BALARSUBRAMANIAN, A.; RANGASWAMI, G. Studies on the influence of foliar nutrient sprays on the root exudation pattern in four crop plants. Plant and Soil, Crawley, v. 30, n. 8, p. 210-220, 1969.

BURKEN, J. G.; SCHNOOR, J. L. Phytoremediation: plant uptake of atrazine and role of root exudates. Journal of Environmental Engineering, London, v. 122, n. 11, p. 958-963, 1996.

CUNNINGHAM, S. D.; ANDERSON, T. A.; SCHWAB, A. P. Phytoremediation of soils contaminated with organic pollutants. Advances in Agronomy, New York, v. 56, n. 1, p. 55-114, 1996.

EDWARDS, C. A. Impact of herbicides on soils ecosystems. Plant Science, Lincoln, v. 8, n. 3, p. 221257, 1989.

FAVERO, C. et al. Modifications in the population of spontaneous plants in the presence of green manure. Pesquisa Agropecuária Brasileira, Brasília, DF, v. 36, n. 11, p. 1355-1362, 2001.

HALE, M. G.; FOY, C. L.; SHAY, F. J. Factors affecting root exudation. Advances in Agronomy, New York, v. 24, n. 7, p. 89-109, 1971.

ISLAM, K. R.; WEIL, R. R. Microwave irradiation of soil for routine measurement of microbial biomass carbon. Biology and Fertility of Soils, Firenze, v. 27, n. 4, p. 408416, 1998.

JENKINSON, D. S.; POWLSON, D. S. The effects of biocidal treatments on metabolism in soil: a method for measuring soil biomass. Soil Biology \& Biochemistry, Davis, v. 8, n. 3, p. 209-213, 1976. 
JENKINSON, D. S.; LADD, J. N. Microbial biomass in soil: measurement and turnover. In: PAUL, E. A.; LADD, J. N. (Eds.). Soil biochemistry. New York: Marcel Deker, 1981. p. $425-471$

KINNEY, C. A.; MANDERNACK, K. W.; MOSIER, A. R. Laboratory investigations into the effects of the pesticides mancozeb, chlorothalonil, and prosulfuron on nitrous oxide and nitric oxide production in fertilized soil. Soil Biology \& Biochemistry, Davis, v. 37, n. 5, p. 837850, 2005.

MOREIRA, F. S. M.; SIQUEIRA, J. O. Microbiologia e bioquímica do solo. Lavras: UFLa, 2002.

NEWMAN, E. I.; WATSON, A. Microbial abundance in the rhizosphere: a computer model. Plant and Soil, Crawley, v. 48, n. 1, p. 17-56, 1977.

PAUL, N. B.; RAO, W. V. B. S. Phosphate-dissolving bacteria in the rhizosphere of some cultivated legumes. Plant and soil, Crawley, v. 35, n. 1, p. 127-132, 1999.

PERKOVICH, B. S. et al. Enhanced mineralization of [14C] atrazine in K. scoparia rhizosferic soil from a pesticide-contaminated site. Pesticide Science, Kyoto, v. 46, n. 4. p. 391-396, 1996.

PERSCHBACHER, P. W. et al. Evaluation of effects of common aerially-applied soybean herbicides and propanyl on the plankton communities of aquaculture ponds. Aquaculture, Arden, v. 157, n. 14, p. 117-122, 1997.

PIRES, F. R. et al. Inferências sobre atividade rizosférica de espécies com potencial para fitorremediação do herbicida tebuthiuron. Revista Brasileira de Ciência do Solo, Viçosa, v. 29, n. 4, p. 627-634, 2005.

PROCÓPIO, S. O. et al . Phytoremediation of soil contaminated with trifloxysulfuron-sodium by Stizolobium aterrimum. Planta daninha, Viçosa, v. 23, n. 4, p. 719724, 2005.

RODRIGUES, B. N.; ALMEIDA, F. S. de. Guia de herbicidas. 5. ed. Londrina: Grafmarke, 2005.

ROVIRA, A. D. Plant root exudates and their influence upon soil microorganisms. In: BAKER, K. F.; SNYDER, W. C. (Eds.). Ecology of soil-borne plant pathogensprelude to biological control. Berkeley: University of California Press, 1965. p. 170-185.

SANTOS, E. A. et al. Fitoestimulação por Stizolobium aterrimum como processo de remediação de solo contaminado com trifloxysulfuron-sodium. Planta daninha, Viçosa, v. 25, n. 2, p. 259-265, 2007.
SANTOS, J. B. Atividade microbiana após aplicação de herbicidas utilizados no cultivo do feijoeiro. 2005. $80 \mathrm{f}$. Tese (Doutorado em Fitotecnia)-Universidade Federal de Viçosa, Viçosa, 2005.

SANTOS, J. B. et al. Action of two herbicides on the microbial activity of soil cultivated with common bean (Phaseolus vulgaris) in conventional-till and no-till systems. Weed Research, Edinburgh, v. 46, n. 4, p. 284289, 2006.

SCRAMIN, S.; SKORUPA, L. A.; MELO, I. S. Utilização de plantas na remediação de solos contaminados por herbicidas: levantamento da flora existente em áreas de cultivo de cana-de-açúcar. In: MELO, I. S. et al. Biodegradação. Jaguariúna: Embrapa Meio Ambiente, 2001. p. 369-371.

STENBERG, B. Monitoring soil quality of arable land: microbiological indicators. Soil and Plant Science, Lincoln, v. 49, n. 1 p. 1-24, 1999.

TAUK, S. M. Biodegradação de resíduos orgânicos no solo. Revista Brasileira de Geociência, São Paulo, v. 20, n. 1-4, p. 299-301, 1990.

VANCE, E. D.; BROOKES, P. C.; JENKINSON, D. S. An extraction method for measuring soil microbial biomass $\mathrm{C}$. Soil Biology \& Biochemistry, Davis, v. 19, n. 6, p. 703707, 1987.

VIVIAN, R. et al. Persistência e lixiviação de ametryn e trifloxysulfuron-sodium em solo cultivado com cana-deaçúcar. Planta daninha, Viçosa, v. 25, n. 1, p. 111-124, 2007.

WARDLE, D. A. Metodologia para quantificação da biomassa microbiana do solo. In: HUNGRIA, M.; ARAÚJO, R. S. (Eds.). Manual de métodos empregados em estudos de microbiologia agrícola. Brasília, DF: Embrapa, 1994. (Documentos, 46).

WILSON, P. C.; WHITWELL, T.; KLAINE, S. J. Metalaxyl and simazine toxicity to and uptake by Typha latifolia. Archives of Environmental Contamination and Toxicology, New York, v. 39, n. 3, p. 282-288, 2000. 